\title{
Nanostructure and Mechanical Properties Studied During Dynamical Straining of Microfibrillar Reinforced HDPE/PA Blends
}

\author{
ZLATAN DENCHEV, ${ }^{1}$ NADYA DENCHEVA, ${ }^{1}$ SÉRGIO S. FUNARI, ${ }^{2}$ MLADEN MOTOVILIN, ${ }^{1}$ TOM SCHUBERT, ${ }^{2}$ NORBERT STRIBECK ${ }^{3}$ \\ ${ }^{1}$ Department of Polymer Engineering, University of Minho, Guimarães 4800-058, Portugal \\ ${ }^{2}$ HASYLAB at DESY, 22603 Hamburg, Germany \\ ${ }^{3}$ Inst. TMC, Department of Chemistry, University of Hamburg, 20146 Hamburg, Germany
}

Received 6 April 2009; revised 17 September 2009; accepted 17 September 2009

DOI: $10.1002 /$ polb.21853

Published online in Wiley InterScience (www.interscience.wiley.com).

\begin{abstract}
Oriented polymer blends whose major component is high-density polyethylene (HDPE) are strained until failure. Two-dimensional (2D) small-angle X-ray scattering (SAXS) patterns monitor the nanostructure evolution, which is related to the macroscopic mechanical evolution. Data evaluation methods for high-precision determination of macroscopic and nanoscopic parameters are presented. The hardest materials exhibit a very inhomogeneous nanodomain structure. During straining, their domains appear to be wedged and inhibit transverse contraction on the nanometer scale. Further components of the blends are polyamide 6 (PA6) or polyamide 12 (PA12) (20$30 \%$ ) and Yparex $^{\circledR} 8102$ (YP) as compatibilizer (0-10\%). Some HDPE/PA6 blends are additionally loaded with commercial nanoclays (Nanomer ${ }^{\circledR}$ or Cloisite ${ }^{\circledR}$ ), the respective amounts being $7.5 \%$ and $5 \%$ with respect to PA. Blending of HDPE with PA12 causes no synergistic effect. In the absence of nanoclay, PA6 and HDPE form a heterogeneous nanostructure with high macroscopic Young's modulus. After addition of YP a rather
\end{abstract}

homogeneous scaffold structure is observed in which some of the PA6 microfibrils and HDPE crystallites appear to be rigidly connected, but the modulus has decreased. Both kinds of nanoclay induce a transition in the HDPE/PA6 blends from a structure without transverse correlation among the microfibrils into a macrolattice with 3D correlations among the HDPE domains from neighboring microfibrils. In the range of extensions between 0.7 and $3.5 \%$, the scattering entities with $3 \mathrm{D}$ correlation show transverse elongation instead of transverse contraction. The process is interpreted as overcoming a correlation barrier executed by the crystallites in an evasion-upon-approaching mechanism. During continued straining, the 3D correlation is reduced or completely removed. (C) 2009 Wiley Periodicals, Inc. J Polym Sci Part B: Polym Phys 48: 237-250, 2010

KEYWORDS: blends; clay; drawing; nanocomposites; SAXS; structure-property relations
INTRODUCTION In polymer engineering, materials with uniaxial orientation are frequently manufactured. These are not only fibers but also extruded strands and pipes. Based on coextrudates from various polymers and nanoparticles, great efforts are made to produce materials with tailored properties. A special class of such materials are the microfibrillar-reinforced composites (MFC). ${ }^{1-6}$ In them, both matrix and reinforcements are obtained in-situ, by transformation of blends of thermoplastic polymers into micro- or nanostructured systems using appropriate mechanical and thermal treatments. The preparation of MFCs comprises three basic steps. First, melt-blending is performed of two or more immiscible polymers. In the polymer blend so formed, the minor phase should always originate from the higher melting material and the major one from the lower melting component. Second, the polymer blend is drawn at temperatures slightly above the glass transition of both blend components leading to their orientation (i.e., fibrillation). Finally, selective liquefaction of the lower melting component is induced. This causes a nearly complete loss of orientation of the major phase, and the isotropic matrix of the composite is created. During this isotropization the temperature is kept below the melting temperature $T_{\mathrm{m} 2}$ of the higher melting and already fibrillated component. Thus, the oriented crystalline structure of the reinforcing fibrils is preserved, and the MFC is formed. Controlling the polymer blend composition and the conditions at each processing step, the diameter of these fibrils, their length and alignment, as well as their adhesion to the matrix can be modified. In such a way, different composites can be produced from similar polymer blends with tensile and flexural properties adjusted to the specific use.

Because the mechanical properties are closely related to failure or fatigue mechanisms on the nanometer scale, monitoring of nanostructure evolution during tensile tests or fatigue experiments is of fundamental relevance. The mechanisms revealed in such studies should help to improve the significance of modeling by advancing the predominantly homogeneous models ${ }^{7}$ 
which are commonly used to predict the properties of polymer materials. Moreover, better understanding of nanostructure evolution mechanisms may immediately gain practical relevance by directing the search for tailored materials.

Static nanostructure can be observed by electron-microscopic methods. Nevertheless, such studies may be impossible for some highly oriented polymer materials, if the sample cannot be adequately fractured or cut. This is the case for the materials presented here. On the other hand, scattering methods do not require special sample preparation. Moreover, they can provide time-resolved data. In particular, the small-angle X-ray scattering (SAXS) can be used to study the nanostructure evolution, although the available instrumentation is enforcing a compromise. Either low-noise patterns are recorded with low time resolution, or noisy patterns with good time-resolution are obtained. For advanced methods of SAXS analysis, we need high-quality two-dimensional (2D) scattering data, and this requirement limits our time resolution to presently $30 \mathrm{~s}$. Moreover, when compared with studies ${ }^{8}$ of elastic materials that break at $200 \%$ elongation $(\varepsilon)$ or more, the study of thermoplastic materials that break at $10 \%$ elongation requires a much higher resolution in $\varepsilon$ to resolve structure evolution. Thus, the presently achievable strain rate $\dot{\varepsilon}$ for thermoplastic materials is by three orders of magnitude lower than the typical industrial strain rates. To our knowledge, the first paper on polymer deformation based on low-noise patterns with an exposure of $15 \mathrm{~s}$ and a cycle time of $30 \mathrm{~s}$ has been published by Chen et al. ${ }^{9}$ In this and similar studies, a stream of high-precision scattering data is obtained. The stream can be considered quasi-continuous, if the variation of nanostructure from frame to frame is small enough. In this case, a survey of the mechanisms of structure evolution is becoming possible.

In this work, we study various MFC precursors, that is, oriented blends before isotropization. As we are aiming at the investigation of materials that fail early at $10 \%$ elongation, even the required determination accuracy of structure parameters becomes a challenge when compared with studies of elastomers. Moreover, because many scattering patterns must be evaluated, automated data analysis is desirable. Corresponding methods are presented both for the high-precision determination of the macroscopic sample elongation, and for the determination of subtle variations of nanostructure parameters.

\section{METHODS}

\section{A Correlation Method to Determine the Macroscopic Elongation from Sample Photos}

To determine the true macroscopic elongation $\varepsilon_{\mathrm{m}}$, the sample with fiducial marks [see Fig. 1(a)] is monitored by a video camera. Fiducial marks have been affixed to the sample by a rubber stamp. In this study, the elongation steps are so small that it is insufficient to measure the mark distances on the screen. Here, we propose a method by which the true macroscopic elongation can be determined automatically and with high precision, if the sample is kept straight and the contrast among the fiducial marks is high enough. Figure 1 demonstrates the method.

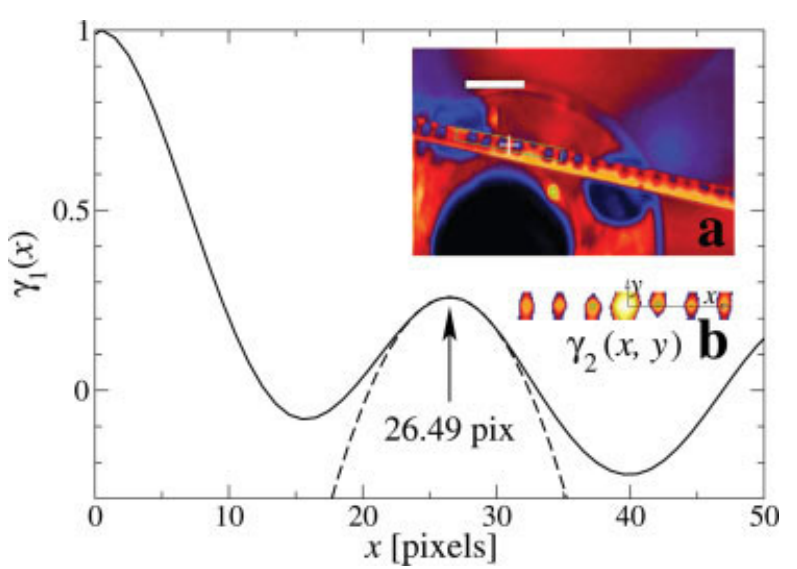

FIGURE 1 True strain from recorded video frames. Inset a: In the first video frame a region of interest (ROI) with fiducial marks is defined. Inset $b$ : From the ROI the 2D correlation function $\gamma_{2}(x, y)$ is computed. Main drawing: The center of the longperiod peak in $\gamma_{1}(x)=\gamma_{2}(x, 0)$ is fitted by a parabola (dashed line) to compute the distance between the fiducial marks. [Color figure can be viewed in the online issue, which is available at www.interscience.wiley.com.]

During the deformation experiment, single frames are grabbed from the video stream and stored as photos. A suitable clock rate is two frames per cycle time of the X-ray detector. Only once the user has to provide some input. It is based on the first image from the TV camera [Fig. 1(a)]. The pseudo-color representation provides good visual contrast. The sample is the straight bar crossing the whole image from the left to the right. The blue spots on the sample are the fiducial marks. The ring-shaped object behind the sample is the flange fixing the entrance window of the vacuum tube between sample and detector. The distance between the fiducial marks is $2 \mathrm{~mm}$ in the shown image.

The center of the X-ray beam on the sample is marked by a cross in the image. Close to this center the user defines a rectangular region of interest (ROI), $\rho_{\mathrm{m}}^{\prime}(x, y)$. In Figure 1(a), this region is bordered by a dashed line. $x$ and $y$ are pixel coordinates in the direction of strain and perpendicular to it, respectively. The same ROI is applied to all video frames of an experiment. The ROI is structured by the fiducial marks running perpendicular to the straining direction. As is known from scattering theory, ${ }^{10}$ the $2 \mathrm{D}$ correlation function

$$
\gamma_{2}(x, y)=\frac{\rho_{\mathrm{m}}^{\star 2}(x, y)}{\rho_{\mathrm{m}}^{\star 2}(0,0)}
$$

of a function $\rho_{\mathrm{m}}(x, y)$ can be computed, with

$$
\rho_{\mathrm{m}}(x, y)=\rho_{\mathrm{m}}^{\prime}(x, y)-\bar{\rho}_{\mathrm{m}}
$$

representing the fluctuation of $\rho_{\mathrm{m}}^{\prime}(x, y)$ about its average $\bar{\rho}_{\mathrm{m}}$, and the autocorrelation being defined by the integral

$$
f^{\star 2}(x, y)=\iint_{-\infty}^{\infty} f(u, v) f(u+x, v+y) \mathrm{d} u \mathrm{~d} v .
$$




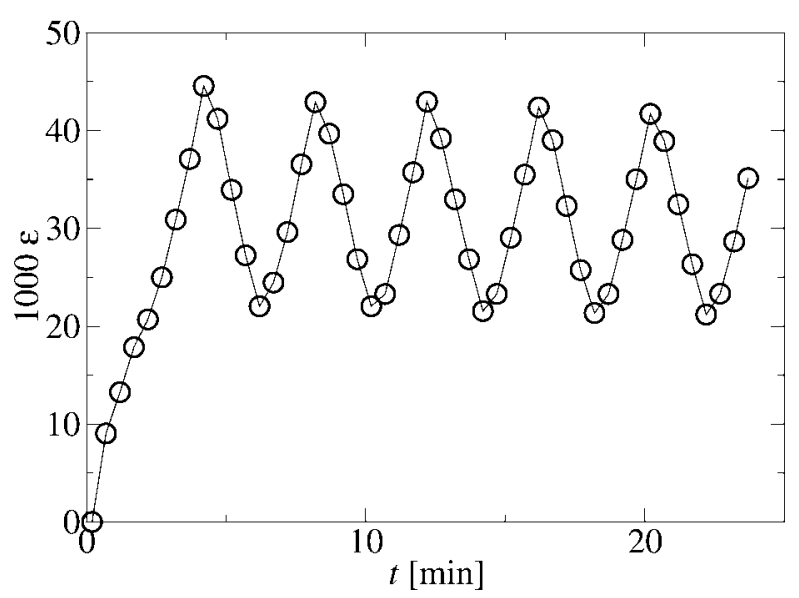

FIGURE 2 Accuracy of the correlation method is demonstrated by the macroscopic elongation $\varepsilon_{\mathrm{m}}(t)$ determined in a load-cycling experiment.

In Figure 1(b), the colored caps demonstrate, where $\gamma_{2}(x, y)$ is positive. Because bar-shaped marks have been affixed to the sample, only the macroscopic elongation $\varepsilon_{\mathrm{m}}$ in straining direction can be extracted from the section $\gamma_{1}(x)=\left\lceil\gamma_{2}(x, y)\right\rceil_{1}(x)$ of $\gamma_{2}$ in straining direction. Figure 1 presents this curve and its analysis. Conforming to the nomenclature of the scattering theory, the first positive peak is the long-period peak that is related to the actual average distance of the fiducial marks, $L_{\mathrm{m}}$. Using the seven data points around the peak maximum, a parabola (dashed line) is fitted, and the position of its vertex is determined (arrow). Thus, in our experiments $L_{\mathrm{m}}$ is determined with an accuracy of 0.01 pixels. Let $L_{\mathrm{m} 0}$ the initial distance between the marks, then the macroscopic elongation is

$$
\varepsilon_{\mathrm{m}}=\frac{L_{\mathrm{m}}}{L_{\mathrm{m} 0}}-1 .
$$

The high accuracy reached is demonstrated in Figure 2 by an $\varepsilon_{\mathrm{m}}(t)$-curve recorded in a load-cycling experiment of one of the MFC samples.

The estimated error is below 0.001. Nevertheless, it increases considerably if the contrast of the bar sequence is low (insufficient illumination) or if the sample is not straight in the beginning. The determination error may also increase after necking has occurred and the sample has begun to slide through the ROI. Finally, it appears worth to mention that in Figure 2 the minima and the maxima of the $\varepsilon_{\mathrm{m}}(t)$ curve had to be approximated, because of a synchronization error between the video capture routine and the cross-bar movement of the tensile tester.

\section{Automated 2D Peak Analysis in Scattering Patterns}

Nanostructure parameters can be extracted either from the measured 2D SAXS patterns $I\left(s_{12}, s_{3}\right)$ in reciprocal space, or in real space from the $\operatorname{CDF} z\left(r_{12}, r_{3}\right)$ (cf. Paragraph "Data evaluation" in Experimental Section). Because narrow peaks of the CDF directly reflect the probability distributions of domain distances, complex multidimensional modeling can be avoided if it is assumed that peak superposition can be neglected.
Thus, to a first approximation nanostructure parameters can be determined directly from peak positions and shapes. In analogy to the method of A Correlation Method to Determine the Macroscopic Elongation from Sample Photos Section, the determination error can be reduced significantly by fitting the peak, and this means that even small variations of nanostructure parameters can be tracked.

For example, if the long-period peak of the matrix material in the CDF is analyzed, then its position on the meridian $\left(r_{3}\right)$ is the long period $L$ that measures the distance in straining direction between neighboring crystallites. Let $L(t)$ the long period at time $t$, and $L_{0}=L(0)$ the long period at the beginning of a deformation experiment, then a nanoscopic elongation

$$
\varepsilon_{\mathrm{nano}}(t)=\frac{L(t)}{L(0)}-1
$$

can be defined. Similarly, if a peak is considered that measures the distance between domains in equatorial $\left(r_{12}\right)$ direction, the

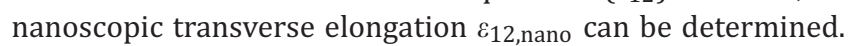

In analogy to the method of A Correlation Method to Determine the Macroscopic Elongation from Sample Photos Section, a ROI must be defined by the user. Inside this ROI, the algorithm searches for the peak. However, now the 2D peak must be fitted to a 2D function. Like in A Correlation Method to Determine the Macroscopic Elongation from Sample Photos Section, a set of measured data points is fed to a regression algorithm. Instead of the seven highest points of a 1D peak, now all those points in a cap are used, for which the intensity is above a user-defined level. The $1 \mathrm{D}$ quadratic polynomial from A Correlation Method to Determine the Macroscopic Elongation from Sample Photos Section is replaced by a 2D (bivariate) polynomial of 2 nd degree. An $n$-th degree bivariate polynomial

$$
f\left(x^{\prime}, y^{\prime}\right)=\sum_{i, j=0}^{n} \mathbf{C}_{i j}^{\prime}\left(x^{\prime}\right)^{i}\left(y^{\prime}\right)^{j}
$$

in the image coordinates $x^{\prime}$ and $y^{\prime}$ is defined by its coefficient matrix $\mathbf{C}^{\prime}$. Let the center of gravity of the fitted polynomial be $\left(x_{\mathrm{g}}^{\prime}, y_{\mathrm{g}}^{\prime}\right)$, then central coordinates are defined by $x=x^{\prime}-x_{\mathrm{g}}^{\prime}$ and $y=y^{\prime}-y_{\mathrm{g}}^{\prime}$. The fitting of 2D data by a bivariate polynomial is a standard method. ${ }^{11}$ In the programming environment PV-WAVE ${ }^{\circledR}, 12$ we utilize for this purpose the library module POLYWARP. PRO and link it into our routine sf_peakfit.pro (free source code available ${ }^{13}$ ). sf_peakfit returns the peak center and the $3 \times 3$ coefficient matrix $\mathbf{C}$ in the central coordinate system. We assume that the profile of the peak in the principal directions $x$ and $y$ can be approximated by Gaussians. Peak widths of Gaussians are characterized by their standard deviations $\sigma$, which are readily expressed in terms of the coefficients of $\mathbf{C}$

$$
\sigma_{x}=\sqrt{\frac{\mathbf{C}_{0,0}}{2 \mathbf{C}_{2,0}}}, \quad \sigma_{y}=\sqrt{\frac{\mathbf{C}_{0,0}}{2 \mathbf{C}_{0,2}}}
$$

from the series expansion of the Gaussians (routines: sf_anapeaks.pro, sf_anapeakso.pro). Here $\sigma_{x}$ and $\sigma_{y}$ 


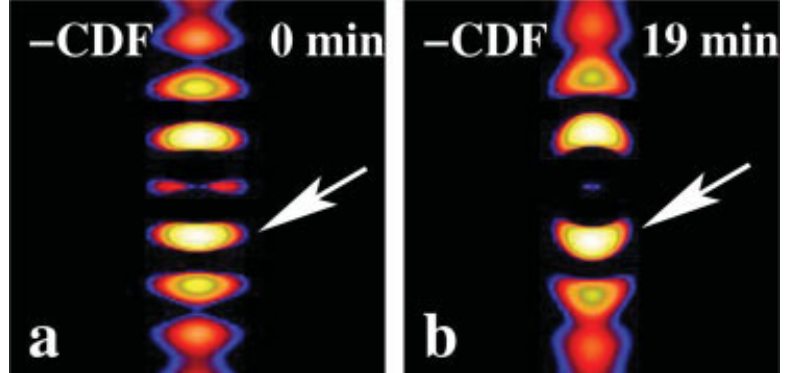

FIGURE 3 Demonstration of peak-shape change from convex (a) to concave (b) in a straining experiment of a HDPE/PA6 blend without compatibilizer (logarithmic intensity scale). [Color figure can be viewed in the online issue, which is available at www.interscience.wiley.com.]

measure the extension of the peak in $x$ - and $y$-direction, respectively. We only use the principal-axis elements of $\mathbf{C}$ for the description of the peak shape. The information on the convexity of the peak that is in the other elements is not yet exploited. Typical concavity is reflected in a banana-shape bending of the peak [cf. Figure 3(b)]. Figure 4 demonstrates the fit of the long-period peak from Figure 3(b) by a bivariate quadratic polynomial. To assure numerical stability of the regression module on digital computers, the maximum intensity in the measured peak data has been normalized to 1 .

Let the $y$-direction be the meridian, and let us consider the analysis of long-period peaks from CDFs, then $\sigma_{y}(t)$ measures the evolution of the width of the long-period distribution inside the sample volume irradiated by the X-ray beam. In analogy to the introduced nanostructure parameter let

$$
D_{\mathrm{L}}(t)=\frac{\sigma_{y}(t)}{\sigma_{y}(0)}-1
$$

be the deviation of the long-period variation from the initial long-period variation. For example, if $D_{\mathrm{L}}(t)=0.05$, then the variation of long periods at time $t$ is by $5 \%$ bigger than before the deformation.

In the studied materials, the CDF long-period peak is constrained in equatorial direction by intensity ridges related to the diameter distribution of microfibrils. Thus, the lateral extension of the peak is a measure of the average diameter of the microfibrils. Then

$$
D_{\mathrm{M}}(t)=\frac{\sigma_{X}(t)}{\sigma_{X}(0)}-1
$$

is the deviation from the initial microfibril diameter. For example, if $D_{\mathrm{M}}(t)=-0.1$, then some average diameter of the microfibrils at time $t$ is by $10 \%$ smaller than before the deformation. In a scattering study, the scattering entities that cause microfibrillar scattering are not only the reinforcing fibrils (polyamide domains, cf. Fig. 11). Additionally, the diameters (i.e., transverse chords) of the HDPE entities contribute to the average $\sigma_{X}$ and to $D_{\mathrm{M}}$. Admittedly, their contribution is only high if the HDPE entities are thin microfibrils, as well.

\section{EXPERIMENTAL}

\section{Materials}

The studied materials are blends made from high-density polyethylene (HDPE) and a polyamide, either polyamide 6 (PA6) or polyamide 12 (PA12). Additionally, some samples contain the commercial compatibilizer Yparex ${ }^{\circledR} 8102$ (YP). Moreover, some HDPE/PA6-blends have been loaded with one of two commercial montmorillonite (MMT) nanoclays. These nanoclays are Cloisite ${ }^{\circledR} 20 \mathrm{~A}$ (by Southern Clay Products) and Nanomer ${ }^{\circledR}$ (by Nanocor), respectively. The nanoclays are delivered in PA6 masterbatches containing up to 20\% MMT.

The blend component HDPE is produced by Borealis [PE VS4531 ${ }^{\circledR}$; density: $0.94 \mathrm{~g} / \mathrm{cm}^{3}$; melt flow index: $0.6 \mathrm{~g} / 10 \mathrm{~min}$ (2.16 kg, $\left.190{ }^{\circ} \mathrm{C}\right)$; melting point by DSC: $133^{\circ} \mathrm{C}$ ]. The PA6 is made by Lanxess [Durethan ${ }^{\circledR}$ B30 S; density: $1.14 \mathrm{~g} / \mathrm{cm}^{3}$; melt volume flow-rate: $110 \mathrm{~cm}^{3} / 10 \mathrm{~min}\left(260{ }^{\circ} \mathrm{C}, 5 \mathrm{~kg}\right.$, ISO 1133); melting point by DSC: $220{ }^{\circ} \mathrm{C}$ ]. The PA12 is produced by EMS-GRIVORY [Grilamid ${ }^{\circledR}$ L25; density: $1.01 \mathrm{~g} / \mathrm{cm}^{3}$; melting point by DSC: $\left.178{ }^{\circ} \mathrm{C} ; M_{\mathrm{w}}=60 \mathrm{~kg} / \mathrm{mol} ; M_{\mathrm{n}}=31 \mathrm{~kg} / \mathrm{mol}\right]$. Yparex $^{\circledR} 8102$ is made by DSM. It is a copolymer of HDPE and maleic anhydride. Its melt flow index is $2.3 \mathrm{~g} / 10 \mathrm{~min}(2.16 \mathrm{~kg}$, $190{ }^{\circ} \mathrm{C}$ ); melting point by DSC: $125{ }^{\circ} \mathrm{C} ; M_{\mathrm{w}}=120 \mathrm{~kg} / \mathrm{mol}$; $M_{\mathrm{n}}=15 \mathrm{~kg} / \mathrm{mol}$.

The investigated materials are highly oriented (fibrillated) polymer blends used as precursors for MFC preparation. The materials without nanoclay have been prepared as follows. Quantities of granulate in the proportions as indicated in Table 1 have been premixed. Each mixture has been meltblended in a laboratory twin-screw extruder. Although the resulting extrudate has been cooled to $12{ }^{\circ} \mathrm{C}$, the first hauloff unit of the extruder line has applied a slight drawing to stabilize the strand cross-section. Further drawing has been performed in the second haul-off unit, after the strand has been heated shortly in a water bath of $97-98{ }^{\circ} \mathrm{C}$. A third haul-off unit has applied the last drawing causing the diameters

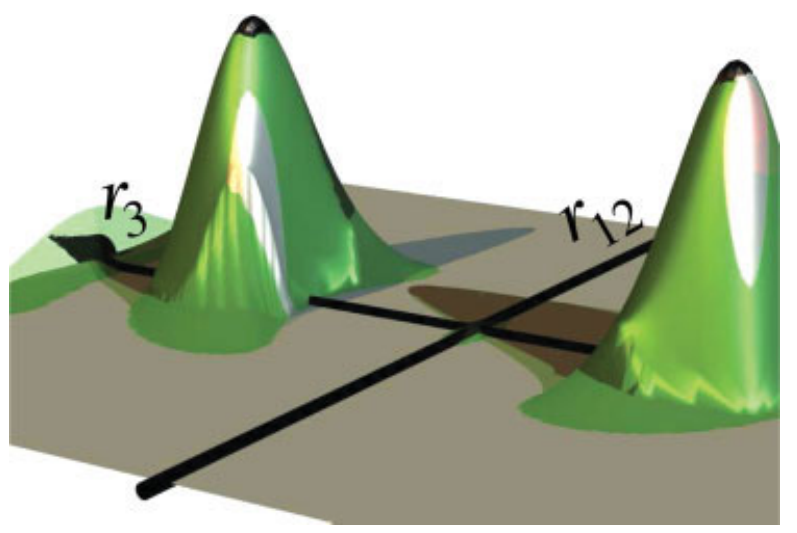

FIGURE 4 Demonstration of bivariate polynomial fitting (linear intensity scale). The measured surface (visualized as glass material) is the concave peak from Figure $3(b)$. The metal-material surface is the fitted polynomial. Its computation considers all points in a cap (in black) that are above $95 \%$ of the peak maximum. [Color figure can be viewed in the online issue, which is available at www.interscience.wiley.com.] 
TABLE 1 Composition (in wt \%) of Oriented Blends without Nanoclay

\begin{tabular}{lllr}
\hline HDPE & PA6 & PA12 & YP \\
65 & & 30 & 5 \\
70 & & 20 & 10 \\
80 & & 20 & 0 \\
65 & & 5 \\
70 & 30 & & 10 \\
80 & 20 & & 0
\end{tabular}

to decrease from $2 \mathrm{~mm}$ (at the extruder die) to about 0.6-0.9 $\mathrm{mm}$ at the end of the extruder line. More details on the extruder line and the processing conditions can be found elsewhere. ${ }^{14}$

The materials with nanoclay (cf. Table 2) have been prepared in the same extruder line. PA6 masterbatches have been diluted with neat PA6 to 5.0 and 7.5 wt \% MMT, respectively, by extrusion blending. Let us call this procedure "pre-blending." After granulation, the preblended PA6 nanocomposites have been mixed with HDPE and YP and drawn as indicated to obtain blends with 20 wt \% of PA6, the latter containing the specified amounts of MMT. In the case of Cloisite, additional oriented samples were prepared by direct blending of weighed amounts of HDPE, PA6/MMT masterbatch, neat PA6, and YP. These materials are labeled "npb" (not preblended). It has been expected that in the preblended Cloisite samples the MMT filler will be concentrated in the PA6 phase only, whereas in the npbmaterials some nanoclay diffusion has been expected also into the HDPE phase.

\section{Straining Environment}

Tensile testing in the X-ray beam is performed using a selfmade extensometer, which has been built by one of us (T.S.). The machine performs symmetric drawing. Signals from load cell and transducer are recorded during the experiment. The sample is monitored by a TV-camera, and video frames are grabbed and stored together with the experimental data. The machine is operated at a cross-bar speed of $0.4 \mathrm{~mm} / \mathrm{min}$. The initial length of the sample between the cross bars is $\sim 30 \mathrm{~mm}$. Measurement of the true macroscopic elongation $\varepsilon_{\mathrm{m}}=\left(\ell-\ell_{0}\right) / \ell_{0}$ is computed from the initial distance, $\ell_{0}$, between fiducial marks and the respective actual distance, $\ell$. The applied high-sensitivity method has been described in A Correlation Method to Determine the Macroscopic Elongation from Sample Photos Section. In the experiments, the true strain rate, $\dot{\varepsilon}_{\mathrm{m}}$, is close to $1.5 \times 10^{-4} \mathrm{~s}^{-1}$.

\section{SAXS Environment}

SAXS is performed at the synchrotron beamline A2 at HASYLAB, Hamburg, Germany. The wavelength of the X-ray beam is $0.15 \mathrm{~nm}$, and the sample-detector distance is $3042 \mathrm{~mm}$. Scattering patterns are collected by a two-dimensional position sensitive marccd 165 detector (mar research, Norderstedt, Germany) operated in $1024 \times 1024$ pixel mode (pixel size: $158.2 \times 158.2 \mu \mathrm{m}^{2}$ ). During the deformation experiments, scattering patterns are recorded every $30 \mathrm{~s}$ with an exposure of $25 \mathrm{~s}$.

\section{Data Evaluation}

The analysis starts from the scattering pattern and turns it into a representation of the nanostructure in real space. The only assumption is presence of a multiphase topology. The result is a multidimensional chord distribution function (CDF), $z(\mathbf{r}) .{ }^{15}$ The method is exemplified in a textbook (Stribeck, ${ }^{10}$ Sect. 8.5.5). For a schematic sketch of the steps of data analysis and the extraction of structural parameters from the CDF see, for example, Figure 2 in Stribeck et al. ${ }^{16}$ The method is extracting the topological information on nanostructure (e.g., a two-phase topology, $\rho(\mathbf{r}) \in\left[\rho_{\text {cryst }}, \rho_{\text {amorph }}\right]$, of phases with distinct densities) from two-dimensional (2D) SAXS patterns with uniaxial symmetry. The resulting CDF is an "edge-enhanced autocorrelation function,"17-20 the autocorrelation of the gradient field, $\nabla \rho(\mathbf{r})$. Thus, as a function of ghost displacement $\mathbf{r}$, the multidimensional $\operatorname{CDF} z(\mathbf{r})$ shows peaks wherever there are domain surface contacts between domains in $\rho(\mathbf{r})$ and in its displaced ghost. The CDF with fiber symmetry in real space, $z\left(r_{12}, r_{3}\right)$, is computed from the fiber-symmetrical SAXS pattern, $I\left(s_{12}, s_{3}\right)$, of multiphase materials. ${ }^{15} \mathbf{s}=\left(s_{12}, s_{3}\right)$ is the scattering vector with its modulus defined by $|\mathbf{s}|=s=$ $(2 / \lambda) \sin \theta$. Here $\lambda$ is the wavelength of radiation, and $2 \theta$ is the scattering angle. In the historical context, the CDF is an extension of Ruland's interface distribution function (IDF) ${ }^{21}$ to the multidimensional case or, in a different view, the Laplacian of Vonk's multidimensional correlation function. ${ }^{22}$

\section{RESULTS AND DISCUSSION}

First, let us present nanostructural mechanisms that are able to explain the evolution of the combined scattering and mechanical data for materials of different composition. After this requested guide to the reader the recorded data themselves are presented and discussed.

\section{HDPE/PA Blends. Mechanisms Explaining the Recorded Structure Evolution}

The nanostructure evolution is different for the different kinds of polyamide (PA12, PA6) used as the reinforcing phase. Related mechanisms that can describe the different evolution of the scattering data are presented in Figure 5.

Striated bars in vertical orientation illustrate the microfibrils made from polyamide. Their intrinsic semicrystalline nanostructure has little scattering effect because of low X-ray

TABLE 2 Composition of Oriented Blends Containing Nanoclay

\begin{tabular}{|c|c|c|c|c|c|}
\hline \multicolumn{3}{|c|}{ wt \% } & \multicolumn{2}{|c|}{ wt \% Clay in PA6 } & \multirow{2}{*}{$\begin{array}{l}\text { Preblended } \\
\text { with Extra PA6 }\end{array}$} \\
\hline HDPE & PA6 & YP & Nanomer & Cloisite & \\
\hline 80 & 20 & & 7.5 & & Yes \\
\hline 77.5 & 20 & 2.5 & 7.5 & & Yes \\
\hline 80 & 20 & & & 5 & Yes \\
\hline 80 & 20 & & & 5 & No \\
\hline 77.5 & 20 & 2.5 & & 5 & Yes \\
\hline 77.5 & 20 & 2.5 & & 5 & No \\
\hline
\end{tabular}




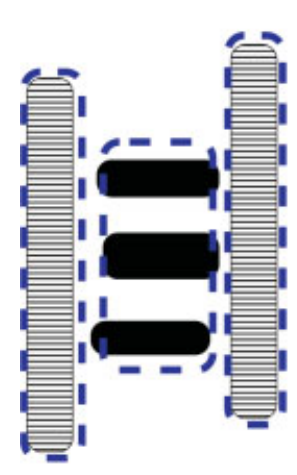

a
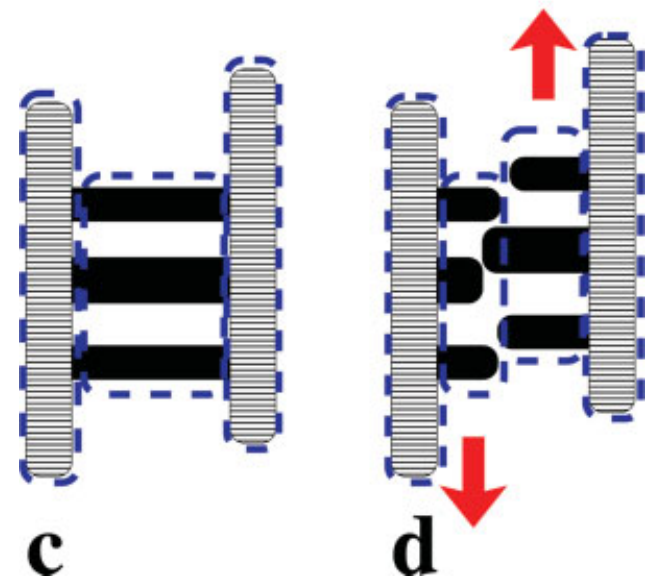

FIGURE 5 Different deformation mechanisms proposed to explain the scattering during extension of blends containing different polyamides. Polyamide: striated vertical bars. HDPE lamellae: black bars. Microfibrillar scattering entities bordered by dashed lines. [Color figure can be viewed in the online issue, which is available at www.interscience.wiley.com.]

contrast and can be neglected. Black bars depict the crystalline domains of the HDPE. Let the gaps be predominantly filled by amorphous HDPE. Dashed curves outline the shapes of the scattering entities that contribute to the microfibrillar scattering of the material. In the SAXS pattern, this scattering is principally reflected in the equatorial streak.

To describe the HDPE/PA12 blends, a model is sufficient in which the two components are not connected. The initial state is shown in Figure 5(a). During straining [Fig. 5(b)], both the macroscopic sample and its nanostructure are affinely deformed in similar manner $\left(\varepsilon_{\text {nano }}(t) \approx \varepsilon_{\mathrm{m}}(t)\right)$. In the experiment, $\varepsilon_{\text {nano }}(t)$ is strongly growing, because the vertical distance between the black bars is increasing. $D_{\mathrm{M}}(t)$ is decreasing moderately, because the scattering entities are subjected to lateral compression.

For the HDPE/PA6/YP blends, the previous model is too simple. Thus, we propose a scaffold-like structure [Fig. 5(c)] that should at least be present in addition to the unconnected nanostructure of Figure 5(a). In the scaffold, the PA6 entities act as stabilizing struts. As the structure is elongated [Fig. 5(d)], the struts prevent an affine extension of the HDPE domain system $\left(\varepsilon_{\text {nano }}(t)<\varepsilon_{\mathrm{m}}(t)\right)$, and the HDPE lamellae themselves are subjected to high load. Failure of the HDPE lamellae causes the average diameter (dashed outline, $D_{\mathrm{M}}(t)$ ) of the microfibrils to decrease considerably.

\section{HDPE/PA6/Nanoclay Blends. Mechanisms Explaining the Recorded Structure Evolution}

Based on the qualitative multidimensional analysis of the CDF (Fig. 8), we propose a mechanism that can explain the observed anomalous evolution of the nanostructure parameters. The model (Fig. 6) is based on the presence of a 3D macrolattice, because this is the fundamental nanostructural difference between the anomalous and the normal materials, that only show uncorrelated microfibrils.

As is generally the case with technical polymers, the nanostructure shows only short-range correlation among domains of varying size and shape. Figure 6(a) sketches an idealized initial structure. We know from the small number of visible CDF peaks that not more than three microfibrils with not more than four domains each are correlated. Moreover, in the sketch the variation of domain shape and size is visualized by gray regions that shall indicate an increased occupation probability of crystalline domains. As this macrolattice is elongated infinitesimally, normal affine behavior (i.e., transverse compression $-\varepsilon_{12 \text {,nano }}$ upon longitudinal elongation $\varepsilon_{\text {nano }}$ ) appears not to be hindered. Moreover, the observed increase of lattice homogeneity does not appear to be unreasonable and has been found in a previous study. ${ }^{23}$ Figure $6(\mathrm{~b})$ sketches the model situation some time later. The central microfibril is under somewhat higher strain, and the correlation towards the neighboring microfibrils is not yet lost. The approaching crystallites (vertical arrows) force their neighbors to move away (horizontal arrows). Under such an evasion mechanism, contact between neighboring crystallites may generate wide microfibrils made from pseudo crystallites (outlined by dashed lines), and this will cause increase of the observed average width of the microfibrils. Moreover, we deduce from the sketch that if this mechanism should hold, then the average distance between the crystallites from different microfibrils
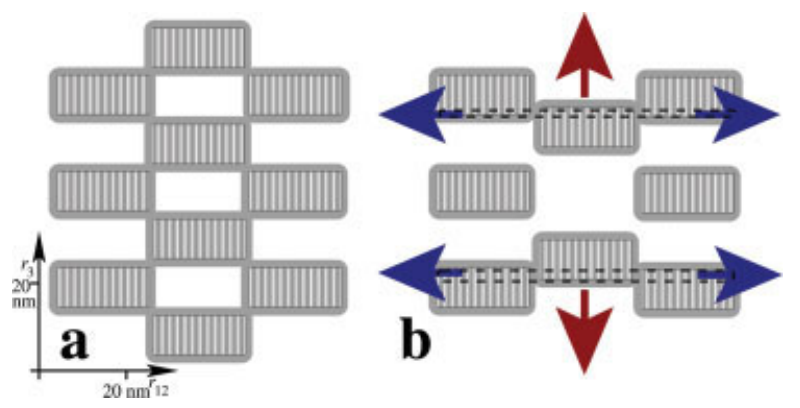

FIGURE 6 Different deformation mechanism explaining the scattering during the extension of HDPE/PA6 blends containing nanoclays. Average domains striated. Gray regions indicating statistical variation of domain sizes. [Color figure can be viewed in the online issue, which is available at www.interscience.wiley.com.] 


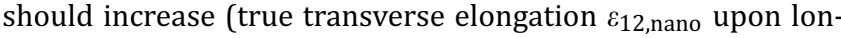
gitudinal elongation $\varepsilon_{\text {nano }}$ ). This effect of transverse elongation can even be measured by monitoring the distance $r_{12}\left(h_{\mathrm{cac}}, t\right)$ of the sandwich-domain peak $h_{\text {cac }}$ from the meridian of the CDF. In Figure 12, this distance is indicated between horizontal arrows.

\section{Nanostructure Evolution: Scattering Patterns HDPE/PA Blends}

Figure 7 presents scattering data of the studied six materials from two different states of the deformation experiment. In each of the six blocks, the left side shows the initial state and the right side the state after $10 \mathrm{~min}$ of straining. This state is reached by all samples without rupture. Comparison shows that the changes are moderate. Thus, a quantitative nanostructure analysis requires a method that permits to determine small variations with sufficient accuracy.

The upper row of each block presents a central detail of the measured SAXS patterns in a logarithmic intensity scaling and pseudo-color. Intensities are normalized with respect to both the incident flux and the sample absorption (variation of irradiated volume). For the presentation, the same intensity value has been mapped to the upper end of the logarithmic scale (pseudo-color: "white"). Scaling overflow has been accepted in several of the images in order to present the features of the different patterns clearly. In the affected patterns, the regions of scaling overflow are restricted to the centers of the scattering patterns (here the color palette is cycled several times). In the CDF images, the scaling is differing from image to image. Here the highest value found in each CDF has been mapped to the upper end of the logarithmic scale.
All SAXS diagrams exhibit both an equatorial streak and a longperiod peak. Peaks exhibiting the linearly elongated shape of Figure $7(\mathrm{a}-\mathrm{e})$ are called layer lines and are typical for a highly oriented microfibrillar system. Thus, even the scattering entities of the HDPE show microfibrillar character. Less pronounced is the orientation of the HDPE microfibrils in the SAXS patterns of the material which contains 30\% PA6 and 5\% YP [Fig. 7(f)]. The pictures in the lower row of each block visualize the nanostructure in CDFs after transformation of the SAXS patterns from reciprocal to real space. Because for all materials all the visible peaks are concentrated in a narrow vertical band about the meridian, we consider it allowed to analyze all materials by the model of a highly oriented microfibrillar system. SAXS monitoring of the melting of these materials have shown ${ }^{14}$ that the dominating long-period peak is vanishing at the melting point of polyethylene. Only after this has happened, the weaker scattering of the semicrystalline polyamide entities becomes visible. Thus, our experiments only probe the semicrystalline structure of the polyethylene and the outer contour of the polyamide microfibrils as far as it contributes to the equatorial streak of the SAXS pattern.

In the SAXS patterns, the distribution functions of the crystalline and the amorphous domains are mapped on subtle variations of the scattering intensity. On the other hand, these functions are the building blocks of the CDFs, where they are clearly visible unless they overlap too badly. Therefore, differences among the nanostructures of the different materials are much more clearly revealed in the CDF than in the SAXS intensity. In Figure 7, the presentation of the CDFs is special, because the absolute value $\left|z\left(r_{12}, r_{3}\right)\right|$ is shown. This presentation ensures that both the positive peaks (domain peaks) and
FIGURE 7 MFC precursor blends from HDPE, two different polyamides (PA12, PA6) and a compatibilizer (YP) in straining experiments. Evolution of nanostructure visualized in $2 \mathrm{D}$ scattering data on logarithmic intensity scales. SAXS patterns: $I\left(s_{12}, s_{3}\right)$ in the region -0.1 $\mathrm{nm}^{-1} \leq s_{12}, s_{3} \leq 0.1 \mathrm{~nm}^{-1}$. CDF patterns: $\left|z\left(r_{12}, r_{3}\right)\right|$ in $-75 \mathrm{~nm}$ $\leq r_{12}, r_{3} \leq 75 \mathrm{~nm}$.
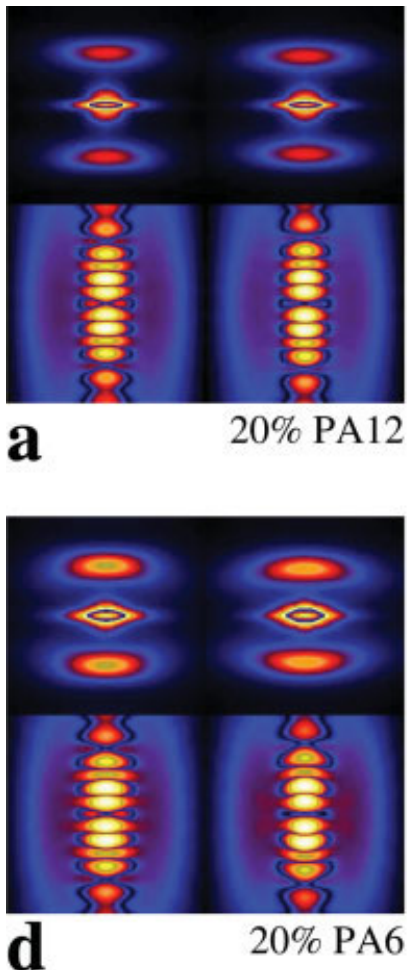

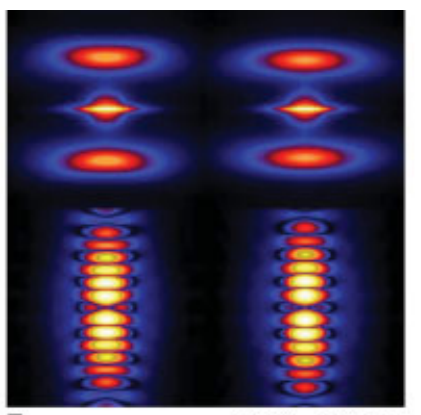

$20 \%$ PA 12

$10 \%$ YP

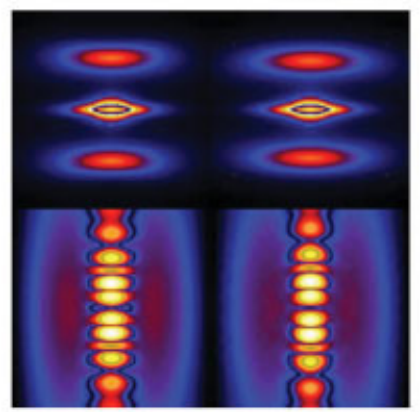

$20 \%$ PA6

$10 \%$ YP

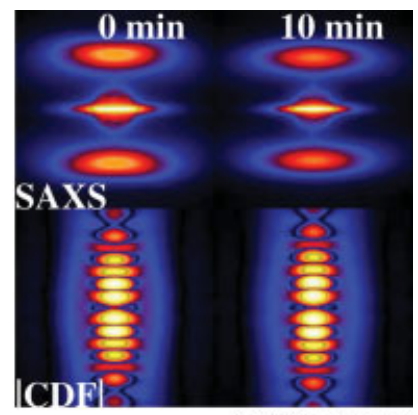

c

$30 \%$ PA12

$5 \%$ YP

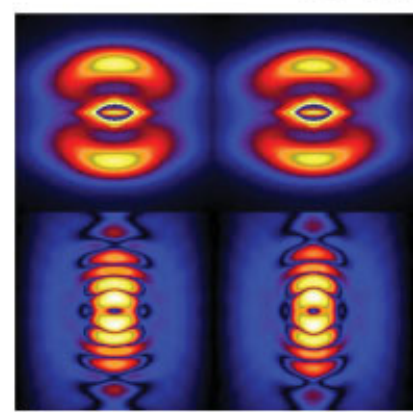

f

$30 \%$ PA6

$5 \%$ YP 
the negative peaks (long-period peaks) are visible at the same time. Because the CDFs show mirror symmetry with respect to the equator plane $r_{12}$, it is sufficient to discuss only the peaks that show up above the equator.

The first peak above the equator describes the distributions $h_{\mathrm{c}}(\mathbf{r})$ and $h_{\mathrm{a}}(\mathbf{r})$ of the crystalline and of the amorphous domains in the HDPE. The individual distributions cannot be discriminated visually, because they are broad and overlap. The next peak is the long-period peak $h_{\mathrm{L}}(\mathbf{r})=h_{\mathrm{ca}}(\mathbf{r})$. The $3 \mathrm{rd}$ peak describes an arrangement composed from three stacked domains $\left(h_{\mathrm{cac}}(\mathbf{r})\right.$ or $\left.h_{\mathrm{aca}}(\mathbf{r})\right)$. The 4 th peak that can be discriminated visually is the second long-period peak made from an arrangement of four domains $\left(h_{2 \mathrm{~L}}(\mathbf{r})=h_{\text {caca }}(\mathbf{r})\right)$. The third long-period peak is already outside the presented region, but still clearly visible. Thus, in all materials atleast three crystalline domains are correlated.

Comparing the CDFs of the different materials, the biggest differences are observed with the $h_{\text {cac }}(\mathbf{r})$ peaks. In the materials without compatibilizer [Fig. $7(\mathrm{a}, \mathrm{d})$ ], these peaks are rather narrow in meridional direction. This means that the height of the corresponding sandwich of domains is the most precisely defined distance between domain surfaces in the stack. Such a special nanostructure is not considered in common models of nanodomain arrangement. It may be explained ${ }^{24,25}$ by directed thickness-growth in coupled twins of nanodomains during crystallization. This exceptional precision of the sandwich height is almost canceled in the material that contains 10 wt \% YP [Fig. 7(b)]. With the HDPE/PA6 blend, the corresponding addition of compatibilizer does not show an effect on the arrangement of the nanodomains [Fig. 7(e)]. On the other hand, here the compatibilizer is considerably increasing the total intensities of the meridional peaks. In the chosen CDF presentation, this leads to the impression as if the intensity ridges parallel to the meridian would disappear. In fact, the apparent effect is simply caused from the adaption of the scaling factor.

All the peak maxima of the semicrystalline structure are on the meridian. This means that the crystalline domains in the HDPE microfibrils are only correlated to each other in meridional direction. There is no correlation in transverse direction among neighboring microfibrils. Thus, the materials without nanoclays do not form a multidimensional macrolattice. ${ }^{26,27}$

\section{HDPE/PA6/Nanoclay Blends}

Figure 8 presents the scattering data of the six studied materials that contain nanoclays. All the CDFs of the unstrained samples are fundamentally different from those of the materials without clay. Although the long-period peaks are still centered on the meridian, here the inseparable domain peaks $h_{\mathrm{c}}+h_{\mathrm{a}}, h_{\text {aca }}+h_{\text {cac }}, h_{\text {cacac }}+h_{\text {acaca }}$ are split into two peaks left and right of the meridian. The 1D microfibrillar nanostructure appears to be replaced by a short-range correlated macrolattice, in which the domains are arranged in a 3D pattern. Figure 9 presents simplified sketches of the two different nanostructures.

Comparing the CDFs of the unstrained materials in Figure 8 with the CDFs in the strained state, different states of
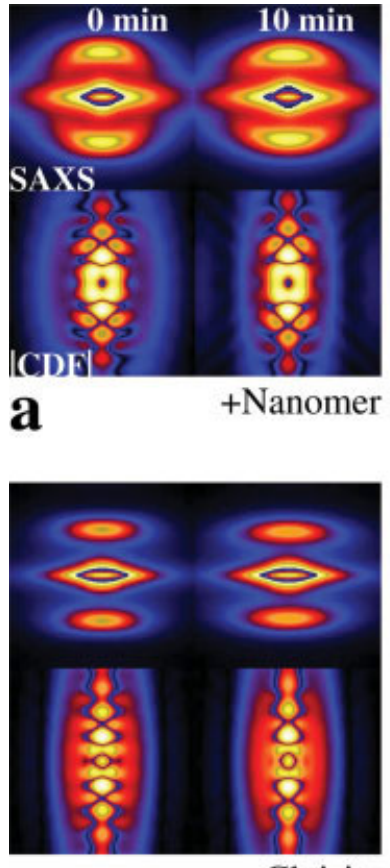

c
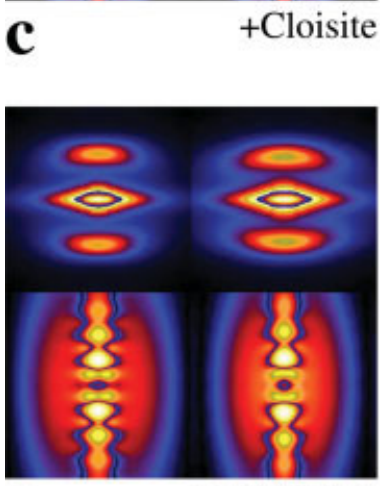

$\mathbf{e}$

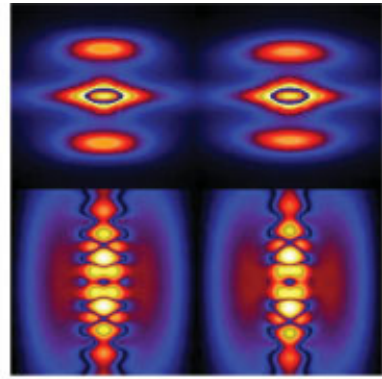

b

+Nanomer

$2.5 \% \mathrm{YP}$

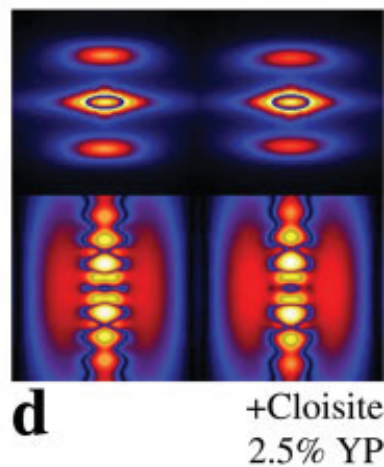

$2.5 \% \mathrm{YP}$

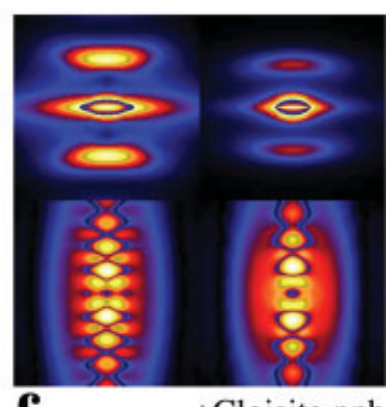

+ Cloisite npb

$2.5 \% \mathrm{YP}$
FIGURE 8 MFC precursor blends from HDPE, PA6, two different nanoclays (Nanomer and Cloisite) and a compatibilizer (YP) in straining experiments. Two different ways of adding Cloisite. Evolution of nanostructure visualized in 2D scattering data on logarithmic intensity scales. SAXS patterns: $I\left(s_{12}, s_{3}\right)$ in the region $-0.1 \mathrm{~nm}^{-1} \leq s_{12}, s_{3} \leq 0.1 \mathrm{~nm}^{-1}$. CDF patterns: $\left|z\left(r_{12}, r_{3}\right)\right|$ in $-75 \mathrm{~nm}$ $\leq r_{12}, r_{3} \leq 75 \mathrm{~nm}$. [Color figure can be viewed in the online issue, which is available at www.interscience.wiley.com.]

conversion into a 1D microfibrillar structure are observed. Thus, straining causes loss of lateral correlation between the microfibrils, and the nanostructure is converted from the 3D macrolattice [Fig. 9(b)] towards the simple arrangement sketched in Figure 9(a). This means that the structure is following the general conversion mechanism described by Peterlin. $^{28}$

The strongest transverse correlation among the microfibrils is shown by the material that contains only preblended nanomer [Fig. 8(a)]. For this material, nanostructure conversion during straining is hardly detected by comparison of the CDFs. Nevertheless, it is found by quantitative analysis. Addition of 


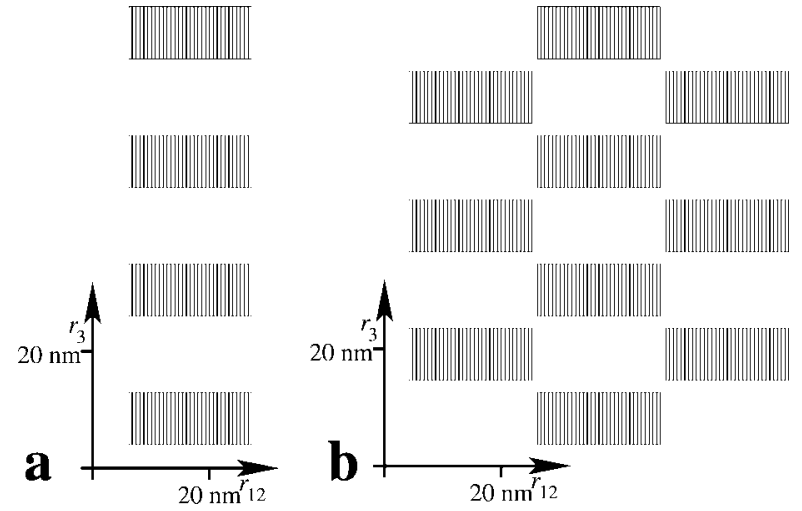

FIGURE 9 Models of the scattering ensembles made from crystalline domains derived from the positions of CDF peaks. (a) HDPE/PA blends without nanoclay. (b) HDPE/PA/nanoclay blends.

compatibilizer [Fig. 8(b)] decreases the transverse correlation among crystallites. The same effect is as well observed with the Cloisite [Fig. 8(c-d)], if the masterbatch and the additional PA6 are preblended. On the other hand, if the masterbatch, the additional PA6 and the HDPE are blended simultaneously [Fig. 8(e-f)], the material containing 2.5 wt \% compatibilizer [Fig. 8(f)] exhibits the most pronounced 3D macrolattice. Subjected to strain, the nanostructure conversion of materials containing Cloisite is proceeding faster than with the materials that contain nanomer. During straining, complete conversion is observed in the test with the normally blended material that contains compatibilizer [Fig. 8(d)].

\section{Nanostructure Evolution: Quantitative Analysis HDPE/PA Blends}

Figure 10 presents the results of a quantitative nanostructure analysis for the blends which do not contain nanoclays. All materials fail by necking close to a clamp. As soon as necking starts, the stress $\sigma$ declines and the sample begins to move through the X-ray beam. In the diagrams the start of this failure process is indicated by an inclined (green) arrow. If the visibility of the sequence of fiducial marks is changing along the sample, the determination of the macroscopic elongation $\varepsilon_{\mathrm{m}}$ (bold dashed lines) becomes less accurate as soon as the sample translation starts. The relatively noisy curve of Figure 10(b) is the result of general poor contrast in the video.

Elongations are illustrated by dashed lines. Bold lines show the macroscopic elongation, $\varepsilon_{\mathrm{m}}$. Thin lines report the nanoscopic elongation $\varepsilon_{\text {nano }}$ of the HDPE matrix. Circular marks indicate regions in which $\varepsilon_{\mathrm{m}} \approx \varepsilon_{\text {nano }}$. All materials reinforced by PA12 exhibit this similarity of macroscopic and nanoscopic deformation. Dashed-dotted lines show $D_{\mathrm{L}}$, the relative variation of the breadth of the long-period distribution. The increase of all curves demonstrates increasing inhomogeneity of the long periods with increasing elongation. Dotted lines show $D_{\mathrm{M}}$, the relative variation of the microfibrillar diameter during the straining process. In all materials, the elongational deformation causes the microfibrils to thin. Variation of the material composition does not cause considerable changes.
This is different with the samples reinforced by PA6 [Fig. 10(df)]. Here an increase of the PA6 content to $30 \%$ causes strong thinning of the microfibrils. In the PA6 blends, the strong transverse decrease is going along with only moderate nanoscopic elongation $\varepsilon_{\text {nano }}$ of the HDPE. An explanation for this finding could be microfibrillation by fracture of crystalline domains of the polyethylene. It may be argued that such microfibrillation should, as well, be detectable in the macroscopic stress-strain curves. In a previous paper some of us have been able to retrieve indications of the macroscopic effect in repetitive load-cycling experiments. There it has been shown ${ }^{8}$ that the stress-strain curve is only somewhat steeper before the disruption of crystallites than afterward. So a corresponding macroscopic slope-change is hard to detect in a simple continuous straining experiment.

Moreover, the diagrams in Figure 10(d-f) demonstrate a considerable difference (vertical arrows) between the two dashed curves. In Figure $10(\mathrm{e}-\mathrm{f})\left(\varepsilon_{\text {nano }}<\varepsilon_{\mathrm{m}}\right)$, the nanoscopic elongation of the HDPE phase is considerably lower than the macroscopic elongation. Similarity is only observed during the initial deformation in Figure 10(f) (circular mark). Such an observed lagging behind of the apparent nanoscopic elongation may be explained by microfibrillation, as well. If one crystallite is broken into two, the number $n$ of crystalline domains in straining direction is increasing to $n+1$ without a sudden increase of sample length $\ell$. The average distance between neighboring crystallites (long period) is computed from the scattering pattern, but after breaking one lamella from the stack it is no longer $L(t)=\ell / n$, but only $L(t)=\ell /(n+1)$. In this way, an apparent retardation of the nanoscopic elongation $\varepsilon_{\text {nano }}(t)=L(t) / L(0)$ can merely be caused by microfibrillation. As a consequence, the long period is only a good measure of nanoscopic elongation, as long as there is no disruption of crystalline domains.

In the 80/20 HDPE/PA6 blend [Figure 10(d)], the nanoscopic elongation of the HDPE microfibrils is considerably longer than the macroscopic elongation $\left(\varepsilon_{\text {nano }}>\varepsilon_{m}\right)$. Although this finding appears to be unreasonable, an indication for a possible mechanism is in the strong increase of $D_{\mathrm{L}}$ [Figure $\left.10(\mathrm{~d})\right]$. This will be discussed in Nanostructure and Mechanical Properties Section.

For the compatibilized HDPE/PA blends, the lagging $\left(\varepsilon_{\text {nano }}<\right.$ $\varepsilon_{\mathrm{m}}$ ) observed with the PA6 blends indicates that the PA6 microfibrils are bonding with the HDPE relatively well, whereas the YP in the blends containing PA12 does not stabilize the HDPE nanostructure. Thus, the deformation mechanisms must be different for blends containing different polyamides. This observation with the compatibilized blends is in agreement with the chemistry of the reaction of YP and PA explained by van Duin, Aussems, and Borggreve. ${ }^{29}$ During the melt mixing, the maleic anhydride from YP reacts with nitrogen atoms from the amide group forming an imide chemical bond across the PA-HDPE interface. In the PA6 repeat unit, there is one $\mathrm{N}$-atom per $5 \mathrm{CH}_{2}$ groups, while in PA12 we have one $\mathrm{N}$ per $11 \mathrm{CH}_{2}$ groups. This makes the probability of a YP-N reaction significantly less probable in the compatibilized HDPE/PA12 blends. Two mechanisms that can 

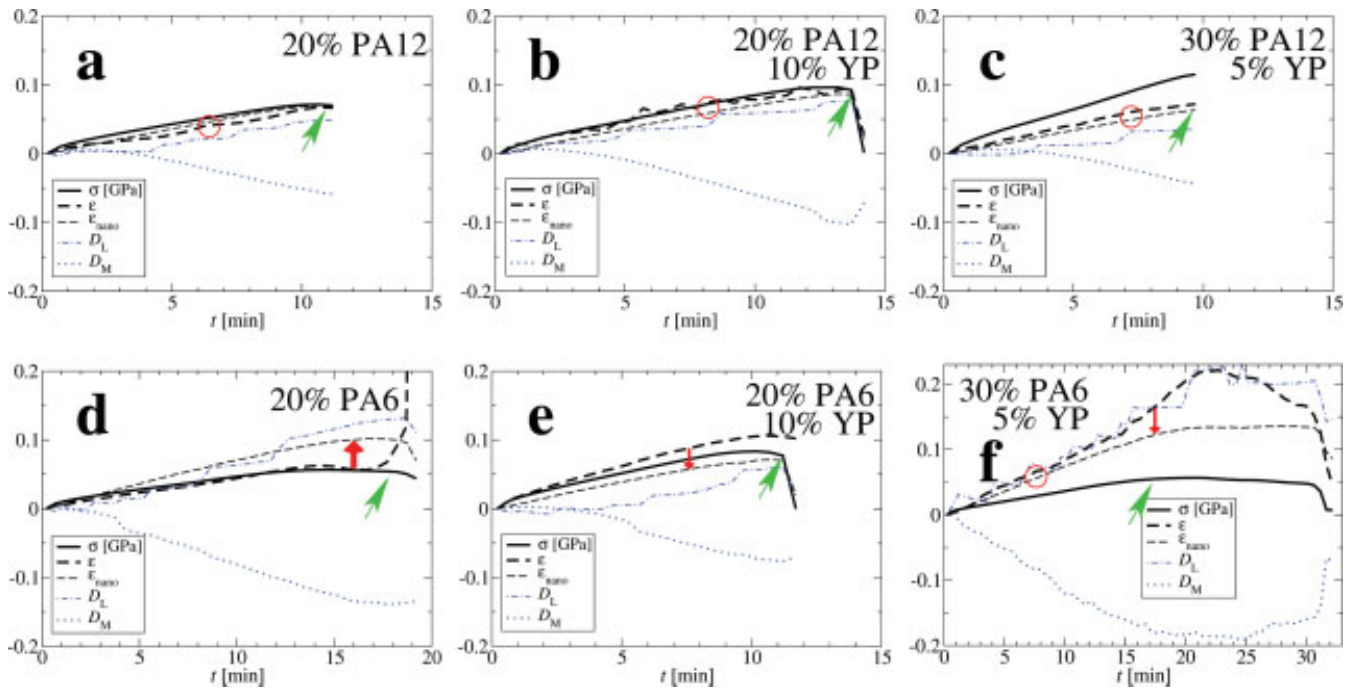

FIGURE 10 MFC precursor blends from HDPE, two different polyamides (PA12, PA6) and a compatibilizer (YP) in tensile tests. Evolution of macroscopic stress and strain $\left(\sigma, \varepsilon_{\mathrm{m}}\right)$ as well as of nanostructure parameters. $\varepsilon_{\text {nano }}$ is the nanoscopic elongation computed from the HDPE long period. $D_{\mathrm{L}}$ is the relative change of the width of the long period distribution. $D_{\mathrm{M}}$ is the relative change of the extension of the microfibrils in transverse direction. [Color figure can be viewed in the online issue, which is available at www.interscience.wiley.com.]

explain the recorded evolution of nanostructure, stress, and strain have been presented in HDPE/PA Blends. Mechanisms Explaining the Recorded Structure Evolution Section.

\section{HDPE/PA6/Nanoclay Blends}

For the HDPE/PA6 blends containing nanoclays, Figure 11 presents the evolution of both the nanostructure parameters and the mechanical data. In general, $\varepsilon_{\text {nano }}(t)<\varepsilon_{\mathrm{m}}(t)$ is observed (downward arrows in the graphs), which is indicative of a mechanically-coupled scaffold structure. One material [Fig. 11(c)] exhibits affine behavior of nanoscopic and macroscopic elongation (circular marks). Another material [Fig. 11(f)] exhibits strongly increased nanoscopic elongation $\varepsilon_{\text {nano }}(t)>\varepsilon_{\mathrm{m}}(t)$ (upward arrow). Compatibilized materials show smoother traces, and the compatibilizer appears to amplify [Fig. 11(a-d)] the lagging behind of $\varepsilon_{\text {nano }}(t)$ for the preblended materials. This is in agreement with the result of the HDPE/PA6 blends without nanoclays, where $\varepsilon_{\text {nano }} / \varepsilon_{\mathrm{m}}$ is highest for the materials containing the highest amount of YP.

With respect to the extracted quantitative structure data, the strongest effect of nanoclay addition is on the shape of the long-period peaks in the CDFs. After the start of the deformation experiment the distances between crystalline HDPE domains ( $D_{\mathrm{L}}$, dashed-dotted lines) grow more uniform if nanoclays are present. Only after a macroscopic elongation of $\varepsilon_{\mathrm{m}} \approx 0.02$ or more is reached, broadening of the long period distribution sets in that is generally typical for the materials without nanoclays. In an intermediate, anomalous regime $\left(0.01 \lesssim \varepsilon_{\mathrm{m}} \lesssim 0.02\right)$ also the average width of the microfibrils $\left(D_{\mathrm{M}}\right.$, dotted lines in Fig. 11$)$ shows an unexpected increase. Such an increase contradicts the common mechanisms of microfibrillation and transverse compression. A possible mechanism that can explain the measured evolution of SAXS, stress, and strain has been presented in HDPE/PA6/Nanoclay Blends. Mechanisms Explaining the Recorded Structure Evolution Section. The mechanism predicts transverse elongation inside the affected macrolattice entities. This effect can be measured by monitoring the distance $r_{12}\left(h_{\mathrm{cac}}, t\right)$ of the sandwich-domain peak from the meridian of the CDF. In Figure 12, this distance is indicated between horizontal arrows. We have chosen this peak from the series of domain peaks, because it is clearly separated from neighboring peaks. Thus, the analysis does not require the peak separation that would be necessary in an analysis of the $h_{\mathrm{c}}$ peak. In the diagram, the dash-dot-dotted curve is showing the measured data for the relative variation

$$
\varepsilon_{12, \text { nano }}=\frac{r_{12}\left(h_{\mathrm{cac}}, t\right)}{r_{12}\left(h_{\mathrm{cac}}, 0\right)}-1 .
$$

Here $r_{12}\left(h_{\text {cac }}, t\right)$ is the $r_{12}$-component of the peak center of the composite peak $h_{\text {aca }}\left(r_{12}, r_{3}\right)+h_{\text {cac }}\left(r_{12}, r_{3}\right)$ at time $t$ as determined by the method described in Automated 2D Peak Analysis in Scattering Patterns Section.

At very low elongation $\left(\varepsilon_{\text {nano }}<0.007\right)$ of the macrolattice in $r_{3}$-direction, $\varepsilon_{12 \text {,nano }}$ becomes negative. Thus, the macrolattice shows transverse contraction typical for the common affine deformation mechanism. Above this level transverse elongation sets in. The maximum is reached at $\varepsilon_{\text {nano }}=0.030$ (cf. Fig. 12 vertical arrow at $t=3 \mathrm{~min}$ ). Simultaneously, the average width of the individual microfibril (cf. $D_{\mathrm{M}}(t)$ in Fig. 12) has increased. This observation is readily explained by considering that some of the crystallites are now in contact and increase the average diameter of crystallites. Above $\varepsilon_{\text {nano }}=0.036$ (i.e., after $5 \mathrm{~min}$ in the experiment), the curve $\varepsilon_{12 \text {,nano }}(t)$ shows a strong decrease. Thus the nanostructure formed by the sets of correlated microfibrils returns to the transverse contraction mechanism. Obviously, the evasion process has lost dominance, most probably because it has destroyed the 3D correlation 

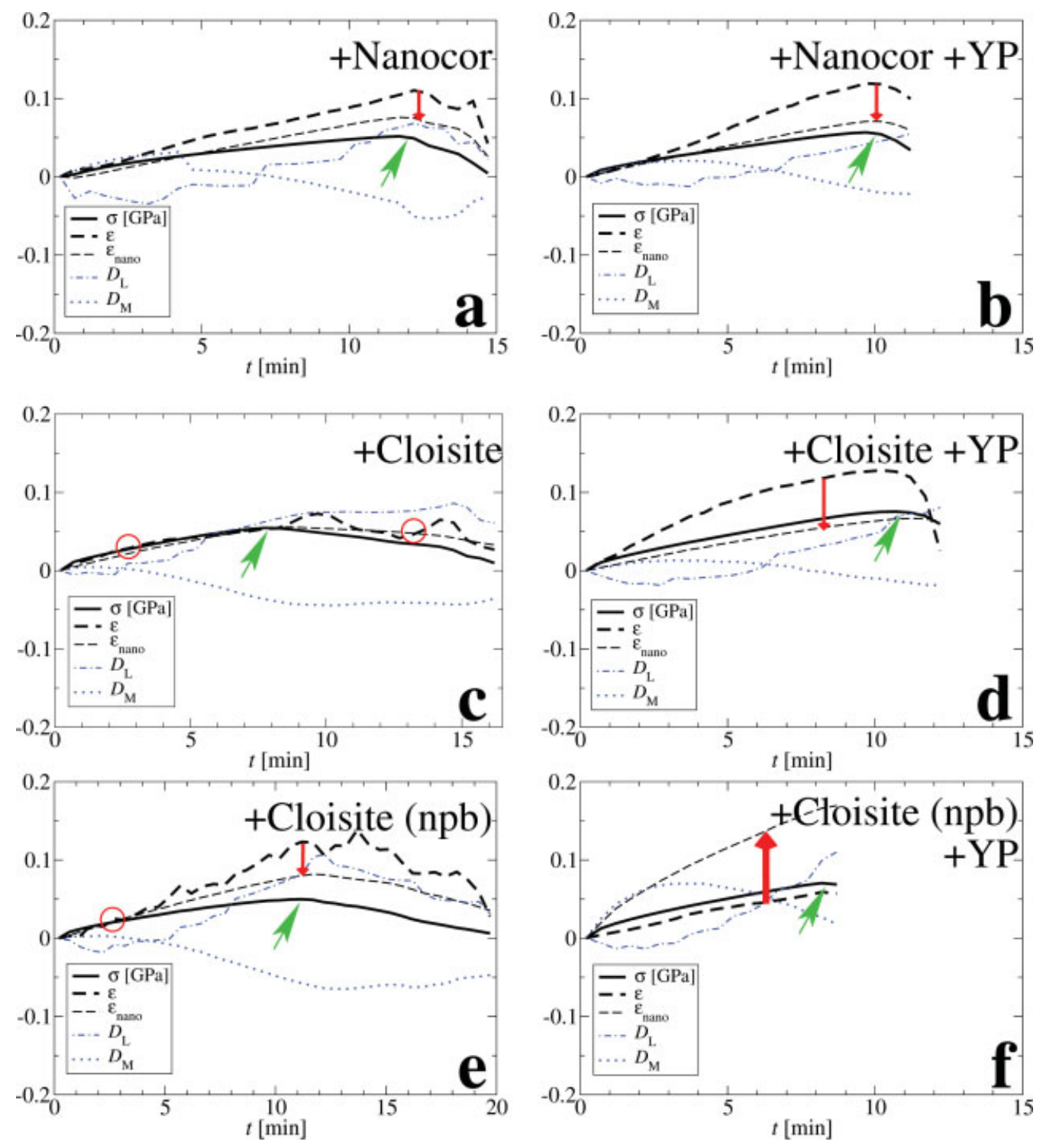

FIGURE 11 MFC precursor blends from HDPE, PA6, two different nanoclays (Nanomer and Cloisite), and a compatibilizer (YP) in tensile tests. Two different ways of adding Cloisite. Evolution of mechanical parameters $\left(\sigma, \varepsilon_{\mathrm{m}}\right)$ and of nanostructure parameters. $\varepsilon_{\text {nano }}$ is the nanoscopic elongation computed from the HDPE long period. $D_{\mathrm{L}}$ is the relative change of the width of the long period distribution. $D_{\mathrm{M}}$ is the relative change of the extension of the microfibrils in transverse direction. [Color figure can be viewed in the online issue, which is available at www.interscience.wiley.com.]

in its macrolattice. If such an evasion mechanism is active, the result should be an inhomogeneous tension distribution inside the material, because the material around the macrolattice regions must be subjected to some super-contraction in order to compensate the transverse elongation of the macrolattice itself. Now the question arises, why the super-contraction does not contribute to the SAXS. As the SAXS has shown the macrolattice is only of short range. So the super-contraction can occur in regions in which the correlations among the crystallites are so low that they do not produce correlation peaks in the CDF.

Admittedly, the average longitudinal elongation $\varepsilon_{\text {nano of only }}$ $3 \%$ will not be sufficient to displace the average crystalline domain by the total amount of its thickness and place it side-by-side to its closest neighbor. On the other hand, the mechanism will become invisible in scattering, if the probing macrolattice is destroyed by loss of lateral correlation. In summary, we propose a superposition of two dynamical statistical mechanisms (evasion coupled to correlation destruction) to explain the finding that the maximum of the addressed transverse elongation is observed at a rather low longitudinal elongation.

Nevertheless, it may be questioned if we have to interpret the observations by transverse elongation of the macrolattice, at all. Explanation by oriented growth of the crystalline layers (stress-induced crystallization) could result in similar shift of 


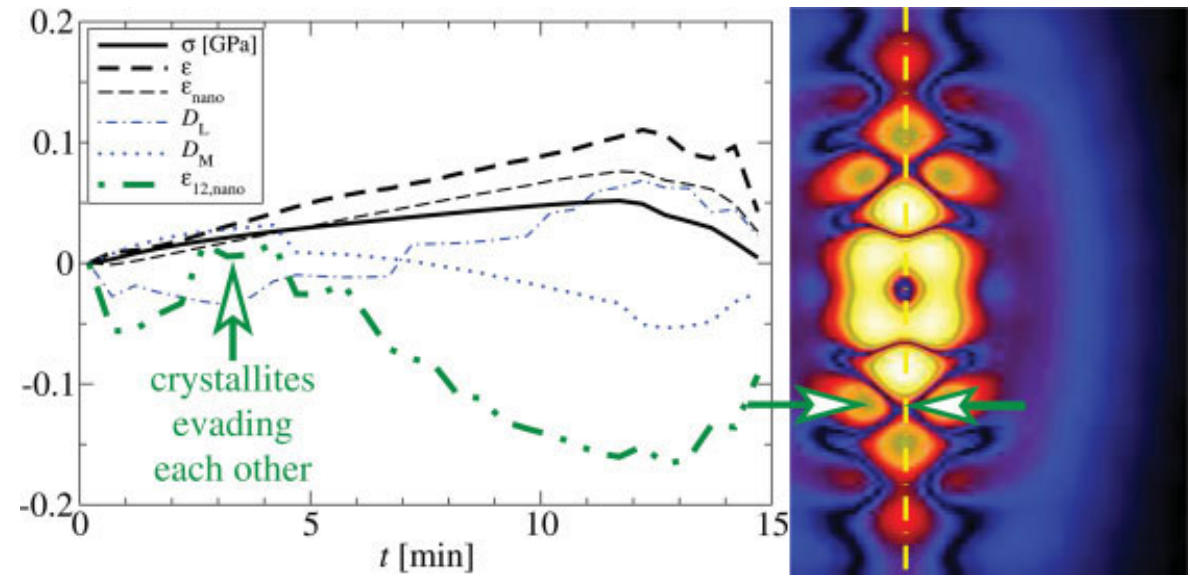

FIGURE 12 Testing the evasion mechanism of crystallites in HDPE/PA6 blends containing nanoclays (here: Nanomer, no compatibilizer). The relative variation of the $r_{12}$-component of the position of the $h_{\text {cac }}\left(r_{12}, r_{3}\right)$-peak (between horizontal arrows) monitors the distance between crystallites from neighboring microfibrils. $\varepsilon_{12, \text { nano }}(t)$ monitors its relative variation. [Color figure can be viewed in the online issue, which is available at www.interscience.wiley.com.] the domain peaks, if preferentially the layers from the two outer microfibrils of the macrolattice entity would grow preferentially away from the central axis of the entity. We believe that this process is less probable than transverse elongation, because the required premises appear to be more restrictive. Another explanation might be deducible from the fact that we are studying cold-drawn strands. It is even possible that the mechanical properties of the strands are not homogeneous. Inside the strands, stress may be frozen in, and the amount of it may vary from shell to core. In this case, the measured "macroscopic strain" would only reflect the elongation of the surface layer with the fiducial marks, but not the elongation of the complete strand.

\section{Measured Mechanical Properties}

The deformation experiments in the synchrotron beam have been carried out dynamically and not in stretch-hold technique. $^{23,30}$ Therefore the actual stress-strain curves can be computed from the recorded data. High-precision determination of the macroscopic elongation $\varepsilon_{\mathrm{m}}$ is accomplished by application of the method presented in A Correlation Method to Determine the Macroscopic Elongation from Sample Photos Section. For the computation of stress the reduction of the fiber diameter during the test is considered assuming constant volume.

\section{HDPE/PA Blends}

Figure 13 presents the macroscopic stress-strain curves of the materials without nanoclays. At elongations of technical relevance $\left(\varepsilon_{\mathrm{m}}>0.01\right)$, the slopes of the curves (Young's modules) differ considerably. For the blends containing PA12 [Fig. 13(a)], high polyamide content (bold dashed line) results in an increased modulus. The linear shape until failure demonstrates ideal Hooke's behavior. Increase of the compatibilizer content (thin dashed line) reduces the modulus at $\varepsilon_{\mathrm{m}} \approx 0.04$ considerably. When compared with the uncompatibilized material (thin solid line), the material exhibits a more rubber-like behavior. The blends reinforced by PA6 show a completely different behavior [Fig. 13(b)]. In general, the stress-strain curves are linear in a broad interval of strain. Here increase of the PA6 fraction to 30 wt \% (bold dashed curve) reduces the modulus considerably. The addition of compatibilizer causes moderate reduction of the modulus (thin dashed curve).

\section{HDPE/PA6/Nanoclay Blends}

Figure 14 presents the macroscopic stress-strain curves of the HDPE/PA6 blends containing nanoclays. Only the dashdot-dotted curve shows considerable increase of the modulus. This material contains compatibilizer, but has not undergone the preblending step (cf. Experimental Section). A moderate
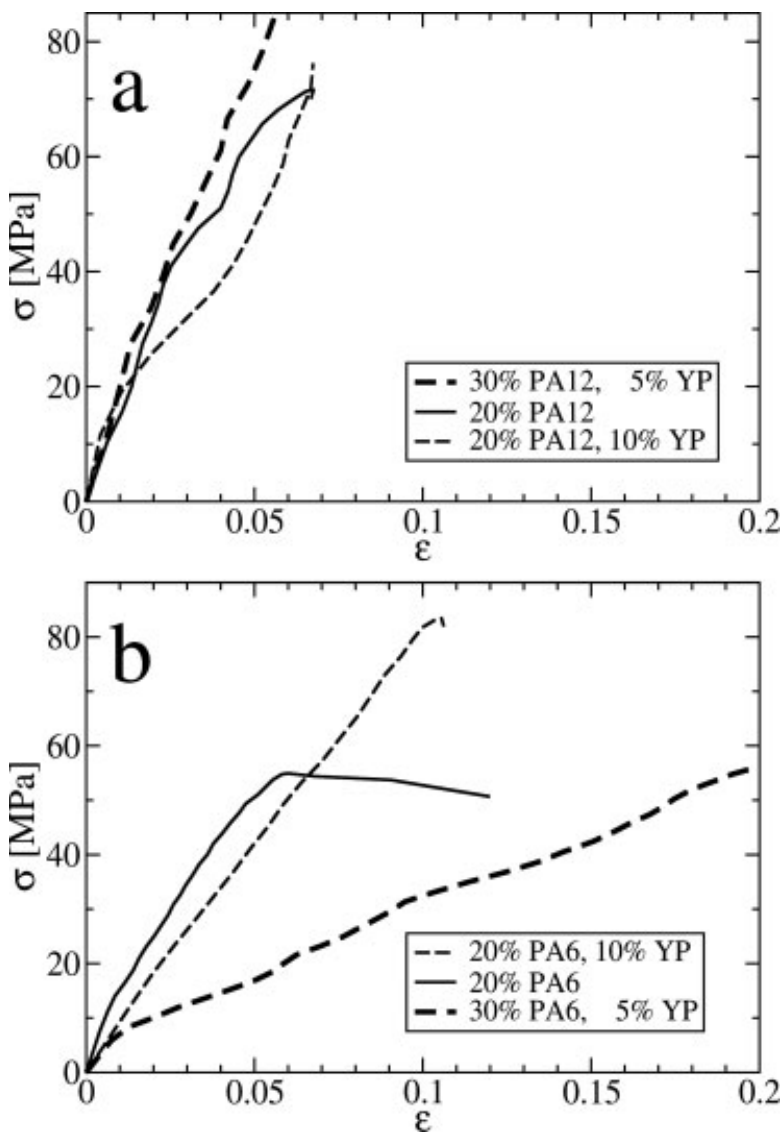

FIGURE 13 Macroscopic stress-strain curves of HDPE/ polyamide blends. 


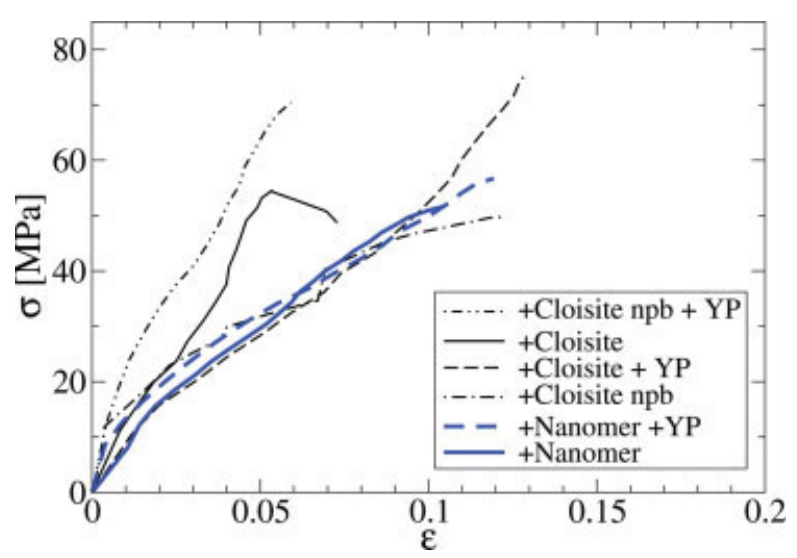

FIGURE 14 Macroscopic stress-strain curves of HDPE/PA6 blends blended with nanoclay (Nanomer, Cloisite) and compatibilizer YP. The label $\mathrm{npb}$ indicates materials for which the extra PA6 has not been preblended with the PA6/Cloisite masterbatch. [Color figure can be viewed in the online issue, which is available at www.interscience.wiley.com.]

increase of the modulus is exhibited (thin solid curve) by the material without YP that contains Cloisite. Here the Cloisite masterbatch and neat PA6 have been preblended to 5 wt \% clay content in the PA6 component.

\section{Nanostructure and Mechanical Properties}

Concerning the nanostructural features and mechanisms that cause a high or a low modulus, some indications can be extracted from the collected results. The two materials with the highest modulus exhibit $\varepsilon_{\text {nano }}(t)>\varepsilon_{\mathrm{m}}(t)$. Additionally, these materials show the strongest increase of $D_{L}$, that is, exceptional inhomogeneity of the domain arrangement in straining direction. Thus, in the hardest materials there are both strongly extended and hardly extended scattering entities. Although, in general, inhomogeneity cannot explain the low macroscopic elongation, it gives an indication. Hardly extended nanoscopic regions that are required to compensate the high value of $\varepsilon_{\text {nano }}$ on the macroscopic scale may contain completely uncorrelated domains that do not contribute to the evaluated long-period peak in the CDF. In this case, nanostructure inhomogeneity is an acceptable explanation for hardening of the material, and the corresponding nanostructure can be imagined as a scaffold, in which domain arrangements from chaotic to regular are present. In the tensile test, the chaotic structure becomes wedged together in space, whereas the regularly arranged regions yield.

An analogous relation between structure and properties has been observed with cold-rolled metals by crystallography and mechanical testing, respectively. Where crystallography reports inhomogenization of the crystal lattice enforced by a distribution of local tensions (strain broadening of crystallographic reflections), on the macroscopic scale "strain hardening"31-33 is observed. Nevertheless, it shall not be concealed that the hardening effect is generally related not to the inhomogeneity, but to a reduced size of the diffracting entities (Hall-Petch effect ${ }^{34,35}$ ).

\section{HDPE/PA Blends}

With the materials containing no nanoclays, reduction of Young's modulus (Fig. 13) is clearly related to the decrease of the HDPE microfibril diameter, $D_{\mathrm{M}}$ (Fig. 10). The stronger the decrease, the lower is the modulus. Reverting this reasoning, suppression of transverse compression, again, appears to be an effective way to harden the material.

The results of the HDPE/PA12 blends indicate that the compatibilizer promotes more regular arrangement of the HDPE crystallites. Thus, the modulus is lowered. The increase of the modulus upon increase of the PA12 fraction is hardly reflected in the nanostructure evolution. Thus, it is considered predominantly an effect of increased PA12 volume fraction.

With the HDPE/PA6 blend with 30\% PA6 it cannot be excluded that both the low modulus (Fig. 13) and the strong decrease of $D_{\mathrm{M}}$ [Fig. 10(f)] are related to less perfect orientation [Fig. 7(f)] of the material during processing. The addition of $10 \%$ compatibilizer has a homogenizing effect on the longitudinal "lattice" inside the HDPE microfibrils and results in a slight decrease of the modulus, but a prolonged linear region in the stress-strain curve. On the other hand, the hard material without compatibilizer exhibits on the nanoscale strong, stepwise increase of the distortions of the longitudinal "lattice" [Fig. 10(d), $\left.D_{\mathrm{L}}\right)$ ]. The end of the linear region in the stress-strain curve is at the beginning of the last step.

\section{HDPE/PA/Nanoclay Blends}

With the HDPE/PA6/nanoclay blends, four samples show almost identical mechanical behavior (Fig. 14). For them comparison to the nanostructure evolution shows $\varepsilon_{\text {nano }}<\varepsilon_{\mathrm{m}}$. The material with the 2 nd highest modulus exhibits $\varepsilon_{\text {nano }} \approx \varepsilon_{\mathrm{m}}$. With the highest-modulus material both $\varepsilon_{\text {nano }}>\varepsilon_{\mathrm{m}}$ is found, and the initial increase of the microfibril diameter, $D_{\mathrm{M}}$, is the highest. Thus, here the hardness of the material appears to be primarily controlled by the inhomogeneity of the nanostructure that causes wedging of the domains in transverse direction and, thus, suppresses transverse contraction.

\section{CONCLUSIONS}

In this study, the information increase by transformation of SAXS patterns into CDFs is clearly demonstrated. By means of the two proposed peak-fitting methods small variations of macroscopic elongation and nanostructure parameters are clearly resolved. In the discussion we have indicated, how relations between properties and nanostructure may be set up. Even though we have advanced the possibilities to study the nanostructure evolution in thermoplastic materials during tensile tests, the representativeness of the results can only be estimated roughly. The method of frequent repetition that is applied in mechanics is not practicable, as long as the evaluation of the corresponding voluminous 2D-SAXS data stays extremely laborious. Therefore, ideal materials for such studies feature high standardization and precisely defined processing parameters.

The authors thank the Hamburg Synchrotron Radiation Laboratory (HASYLAB) for beam time granted in the frame of project II-04-039. Part of this work has been supported 
by the 7th framework program of the European Union (Project NANOTOUGH FP7-NMP-2007-LARGE). Z. D. is grateful for the tenure of a sabbatical grant awarded by the Fundação para a Ciência e Tecnologia, Portugal (grant No SFRH/BSAB/812/2008) and for the hospitality of the Institute for Technical and Macromolecular Chemistry, Hamburg.

\section{REFERENCES AND NOTES}

1 Evstatiev, M.; Fakirov, S. Polymer 1992, 33, 877-880.

2 Evstatiev, M.; Nicolov, N.; Fakirov, S. Polymer 1996, 37, 44554463.

3 Fakirov, S.; Stribeck, N.; Apostolov, A. A.; Denchev, Z.; Krasteva B.; Evstatiev, M.; Friedrich, K. J Macromol Sci Phys 2001, 40, 935-957.

4 Schmidt, P.; Baldrian, J.; Ščudla, J.; Dybal, J.; Raab, M.; Eichhorn, K.-J. Polymer 2001, 41, 5321-5326.

5 Li, Z.-M.; Yang, M.-B.; Feng, J.-M.; Yang, W.; Huang, R. Mater Res Bull 2002, 37, 2185-2197.

6 Denchev, Z. Z.; Dencheva, N. V. Polym Int 2008, 57, 11-22.

7 Bicerano, J. Prediction of Polymer Properties, 3rd ed.; CRC Press: New York, 2002.

8 Stribeck, N.; Nöchel, U.; Funari, S. S.; Schubert, T.; Timmann, A. Macromol Chem Phys 2008, 209, 1992-2002.

9 Chen, X.; Yoon, K.; Burger, C.; Sics, I.; Fang, D.; Hsiao, B. S.; Chu, B. Macromolecules 2005, 38, 3883-3893.

10 Stribeck, N. X-Ray Scattering of Soft Matter; Springer: Heidelberg, New York, 2007.

11 Hall, E. L. Computer Image Processing and Recognition; Academic Press: London, 1980.

12 VNI, “PV-WAVE manuals," V 7.5, Houston, TX, USA, 2007.

13 Stribeck, N. Downloads, available at: http://www.chemie.unihamburg.de/tmc/stribeck/dl, 2008.

14 Dencheva, N.; Oliveira, M. J.; Carneiro, O. S.; Pouzada, A. S.; Denchev, Z. J Appl Polym Sci 2009, submitted for publication.
15 Stribeck, N. J Appl Cryst 2001, 34, 496-503.

16 Stribeck, N.; Almendarez Camarillo, A.; Nöchel, U.; Schroer, C.; Kuhlmann, M.; Roth, S. V.; Gehrke, R.; Bayer, R. K. Macromol Chem Phys 2006, 207, 1239-1249.

17 Debye, P.; Bueche, A. M. J Appl Phys 1949, 20, 518-525.

18 Porod, G. Colloid Polym Sci 1951, 124, 83-114.

19 Vonk, C. G. J Appl Cryst 1973, 6, 81-86.

20 Baltá Calleja, F. J.; Vonk, C. G. X-Ray Scattering of Synthetic Polymers; Elsevier: Amsterdam, 1989.

21 Ruland, W. Colloid Polym Sci 1977, 255, 417-427.

22 Vonk, C. G. Colloid Polym Sci 1979, 257, 1021-1032.

23 Stribeck, N.; Nöchel, U.; Funari, S. S.; Schubert, T. J Polym Sci Part B: Polym Phys 2008, 46, 721-726.

24 Stribeck, N.; Bayer, R.; Bösecke, P.; Almendarez Camarillo, A. Polymer 2005, 46, 2579-2583.

25 Stribeck, N.; Bösecke, P.; Bayer, R.; Almendarez Camarillo, A. Progr Colloid Polym Sci 2005, 130, 127-139.

26 Fronk, W.; Wilke, W. Colloid Polym Sci 1985, 263, 97-108.

27 Stribeck, N.; Androsch, R.; Funari, S. S. Macromol Chem Phys 2003, 204, 1202-1216.

28 Peterlin, A. Text Res J 1972, 42, 20-30.

29 van Duin, M.; Aussems, M.; Borggreve, R. J. M. J Polym Sci Part A: Polym Chem 1998, 36, 179-188.

30 Wu, J.; Schultz, J. M.; Yeh, F.; Hsiao, B. S.; Chu, B. Macromolecules 2000, 33, 1765-1777.

31 Dehlinger, U.; Kochendörfer, A. Z. Kristallograf 1939, 101, 134-148.

32 Kochendörfer, A. Z. Kristallograf 1944, 105, 393-438.

33 Warren, B. E.; Averbach, B. L. J Appl Phys 1950, 21, 595-599.

34 Chokshi, A. H.; Rosen, A.; Karch, J.; Gleiter, H. Scr Metall 1989, 23, 1679-1683.

35 Weertman, J. R. Mater Sci Eng A 1993, A166, 161-167. 